\title{
Cross-Field Motion of Plasma Blobs in an Open Magnetic Field Line Configuration
}

\author{
C. Theiler, ${ }^{1}$ I. Furno, ${ }^{1}$ P. Ricci, ${ }^{1}$ A. Fasoli, ${ }^{1}$ B. Labit, ${ }^{1}$ S. H. Müller, ${ }^{1,2}$ and G. Plyushchev ${ }^{1}$ \\ ${ }^{1}$ Centre de Recherches en Physique des Plasmas, Ecole Polytechnique Fédérale de Lausanne (EPFL), \\ Association EURATOM-Confédération Suisse, CH-1015 Lausanne, Switzerland \\ ${ }^{2}$ Center for Energy Research, University of California, San Diego, La Jolla, California 92093, USA
}

(Received 16 December 2008; published 3 August 2009)

\begin{abstract}
The radial propagation of blobs generated from plasma instabilities is investigated in an open magnetic field line configuration. Blob cross-field velocities and sizes are obtained from internal probe measurements using pattern recognition. By varying the ion mass, the normalized vertical blob scale $\tilde{a}$ is scanned from $\tilde{a}<1$ to $\tilde{a}>1$. An analytical expression for the blob velocity including cross-field ion polarization currents, parallel currents to the sheath, and ion-neutral collisions is derived and shows good quantitative agreement with the experimental data. In agreement with previous theoretical studies, this scaling shows that, for $\tilde{a}<1$, the blob velocity is limited by cross-field ion polarization currents, while for $\tilde{a}>1$ it is limited by parallel currents to the sheath.
\end{abstract}

PACS numbers: 52.25.Fi, 52.25.Ya, 52.35.Ra

Plasma particles and energy can efficiently be convected across a confining magnetic field in the form of blobs, coherent structures of enhanced plasma density relative to the background plasma. Blobs are filaments, as they typically extend much further along the magnetic field than perpendicular to it. They are measured near the edge of virtually all magnetized laboratory plasmas, including tokamaks, stellarators, reversed field pinches, simple magnetized tori, and linear devices [1-5].

In the absence of magnetic fluctuations, blob propagation can qualitatively be understood as follows [6]: charge dependent drifts, such as those generated by magnetic field gradients and curvature, lead to cross-field currents and, due to the blob spatial inhomogeneity across the magnetic field, to charge separation. The resulting electric field inside the blob gives rise to an $\mathbf{E} \times \mathbf{B}$ motion. The magnitude of the cross-field velocity depends on the available current paths to damp charge separation. If the damping results from parallel currents to the sheath, an analytical solution for the blob motion exists [6]. This predicts a blob velocity $\propto 1 / \tilde{a}^{2}$, where $\tilde{a}$ is the normalized vertical blob size [see Eq. (3) for definition]. If cross-field ion polarization currents are considered in the absence of parallel currents, a blob initially at rest accelerates and reaches an approximately constant velocity $\propto \sqrt{\tilde{a}}[7,8]$. Numerical simulations of blob motion that include both parallel currents to the sheath and ion polarization currents predict that coherent blob motion is limited to $\tilde{a} \sim 1$ and that parallel currents become dominant for $\tilde{a}>1$ [9-11].

Blob motion is extensively studied in the scrape-off layer (SOL) of fusion plasmas, because of its importance for the design of burning plasma experiments and future fusion reactors $[12,13]$. However, a conclusive comparison with theoretical models is hampered by limited diagnostic accessibility in these devices, uncertainties in the relevant parameters, and the large number of effects that can arise due to the complicated geometry [14].
In this Letter, we investigate plasma blob motion in the TORPEX [15] toroidal device, a simple open magnetic field line geometry with constant curvature along the field lines. In TORPEX, internal measurements over the entire plasma cross section are possible, owing to relatively low plasma temperatures and densities. Cross-field sizes and velocities are obtained for a large number of blobs from a 2D array of Langmuir probes (LPs) using pattern recognition [16]. By varying the ion mass, we find blob sizes in the range $0.15 \lessgtr \tilde{a} \lesssim 1.75$. While the $\sqrt{\tilde{a}}$ scaling provides a good estimate of the measured radial blob velocity for $\tilde{a}<1$, strong deviations are found for larger blobs, demonstrating the importance of parallel currents. We derive an analytical expression for the blob velocity that agrees with the whole data set.

The experimental setup is similar to the ones in [2,3]: a vertical magnetic field of $1.6 \mathrm{mT}$ is superimposed on a toroidal field of $76 \mathrm{mT}$, creating helical field lines. The plasma source is located on the high-field side, i.e., on the inner side of the device cross section. Blobs form from an interchange wave and propagate radially through a sourcefree region, exhibiting universal statistical properties similar to the SOL of fusion plasmas [17] and contributing significantly to cross-field particle transport $[2,18]$. Studies on blob formation in this setup have revealed that blobs form from radially extending positive crests of the wave [2] that get sheared apart by the $\mathbf{E} \times \mathbf{B}$ flow [3]. The radial elongation of the wave is attributed to a decrease in the radial pressure scale length $[3,19]$.

For the present experiments, a steel limiter was inserted in the blob region, i.e., in the outer half of the cross section, as schematically shown in Fig. 1(a). This results in a region on the low-field side characterized by a nearly constant connection length $L \approx 2 \pi R, R=1 \mathrm{~m}$ being the major radius, and near-perpendicular incidence of the magnetic field lines on the material surface. The latter avoids complicating effects such as the contribution of the electron 

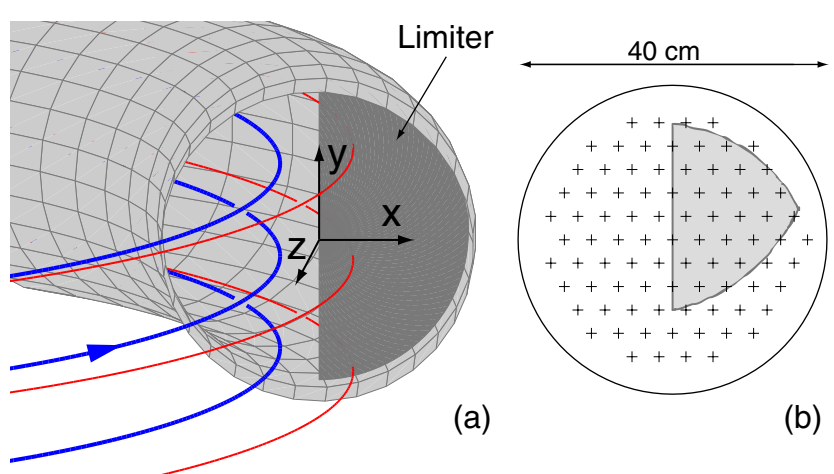

(a)

(b)

FIG. 1 (color online). (a) Sketch of the experimental setup with two examples of helical magnetic field lines. The thick blue line lies in the main plasma region and does $\sim 3$ turns before intercepting the vacuum vessel. The thin red line lies in the blob region and intercepts the limiter plate after one turn. (b) Arrangement of the LP tips (crosses) of the 2D array. The shaded area indicates the region where both field line ends are connected to the limiter.

diamagnetic current to the blob parallel current, expected for small incidence angles between magnetic field lines and wall [20]. In order to significantly vary $\tilde{a}$, discharges in four different gases are performed $\left(\mathrm{H}_{2}, \mathrm{He}, \mathrm{Ne}, \mathrm{Ar}\right)$. In Figs. 2(a)-2(d), we show snapshots of the electron density obtained in these plasmas. The time averaged profiles (white solid contours) peak on the high-field side. Strong fluctuations around the average profiles and the radial movement of blobs are apparent.

Blob propagation is investigated using a $2 \mathrm{D}$ LP array [21], toroidally displaced by $97^{\circ}$ from the limiter. This array comprises 86 LPs with $3.5 \mathrm{~cm}$ pin separation [see sketch in Fig. 1(b)] and provides the evolution of the ion saturation current $\left(I_{\mathrm{sat}}\right)$ with a temporal resolution of $4 \mu \mathrm{s}$. Blobs are identified by pattern recognition [16] and their sizes are determined as follows. During the first four time frames, we evaluate the $y$ profile of $I_{\text {sat }}$ over a vertical cut through the center of the blob. Examples are shown in Figs. 2(e) and 2(f). We then subtract the background density, taken as the average of the nearest local minima located above and below the position of the maximum of $I_{\text {sat. }}$. The vertical blob size $a$ is defined as the average of the HWHM of these four $I_{\text {sat }} y$ profiles. To assess the size resolution achieved by this procedure, we apply the analysis to synthetic Gaussian shaped blobs mapped to the LP grid. We find that structure sizes are resolved with an average error $\lesssim 20 \%$ as long as $a \gtrsim 1.5 \mathrm{~cm}$. Structures with $a \lesssim 1.5 \mathrm{~cm}$ are not detected by pattern recognition, as long as they do not stay close to one LP tip during their lifetime. In the following, we consider structures that exist for at least 10 time frames $(36 \mu \mathrm{s})$ for $\mathrm{H}_{2}, \mathrm{He}$, and $\mathrm{Ne}$ and 20 time frames $(76 \mu \mathrm{s})$ for the significantly slower blobs in Ar, respectively. The selected blobs then typically move by at least one tip spacing during their trajectory, which avoids the detection of blobs with $a \lesssim 1.5 \mathrm{~cm}$. We additionally require that blobs do not undergo merging or splitting [16]
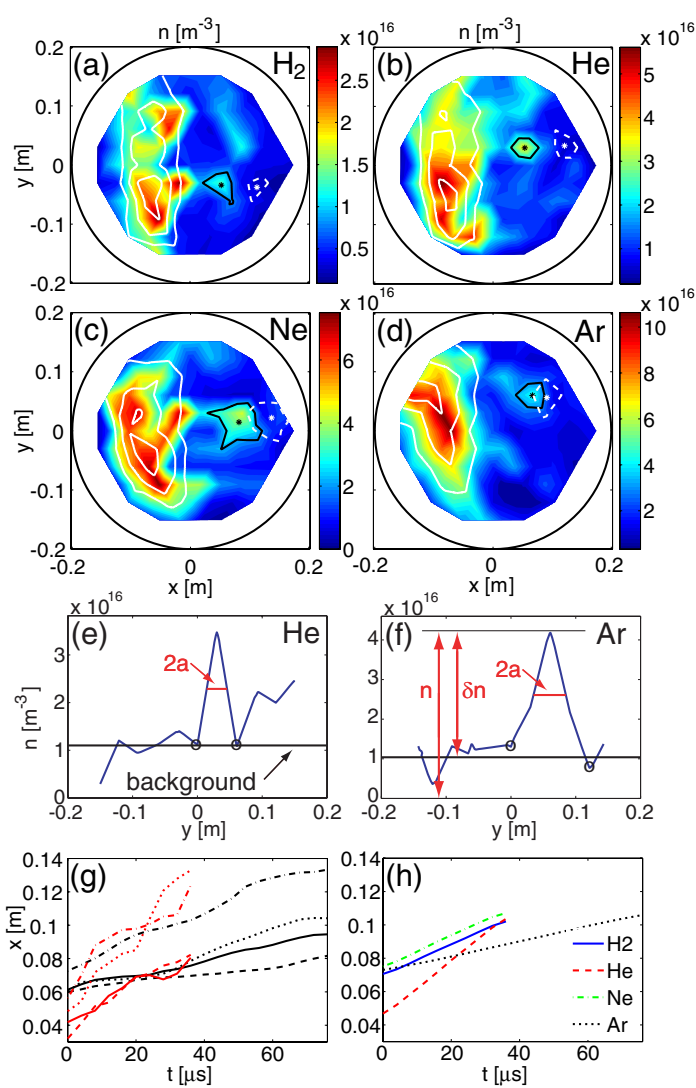

FIG. 2 (color online). (a)-(d) Snapshots of electron density $n$ in the different plasmas. Time averaged profiles are indicated by white contours. A blob as well as its location $36 \mu$ s later (76 $\mu \mathrm{s}$ in the case of Ar) is shown on the low-field side. $n$ is obtained from $I_{\text {sat }}$ by assuming $T_{e}=8,14,12$, and $7 \mathrm{eV}$, respectively, corresponding to the peak electron temperatures measured with swept LPs in the four plasmas. (e),(f) Vertical cut through the He and the Ar blob in (b) and (d), together with the evaluated quantities $a, \delta n$, and $n$. Blob radial position as a function of time (g) for selected blobs in He [gray (red)] and Ar (black), and (h) averaged over all blobs in a given gas.

during that time, that they travel radially at least to $x_{\min }=$ $4 \mathrm{~cm}$, and that their trajectories lie mostly in the shaded area of Fig. 1(b). Further, we focus on structures that propagate almost monotonically outwards, i.e., for which $\Delta x_{i}<0$ is not allowed for two subsequent steps, where $\Delta x_{i}$ is the radial displacement of the blob between frame $i$ and frame $i+1$. The latter condition excludes between $\sim 35 \%(\mathrm{He})$ and $\sim 55 \%(\mathrm{Ne})$ of the detected structures. These are mostly structures that, after detaching from the wave crest, do not propagate radially and do not contribute to perpendicular transport. Examples of blobs satisfying the selection criteria are indicated in Figs. 2(a)-2(d).

We have selected between $395(\mathrm{Ar})$ and $1185\left(\mathrm{H}_{2}\right)$ blobs. In Fig. 2, we plot the radial position versus time for individual blobs $(g)$ and on average $(h)$. We note that the fluctuations of the instantaneous velocity during individual trajectories can be large $(g)$. This can be explained by the finite spatial resolution of the grid. Trajectories in $(h)$ where finite grid effects are averaged out show relatively 
small fluctuations $(\leq 30 \%)$. Therefore, we define the velocity of an individual blob as the mean velocity over its entire trajectory. In Fig. 3, we plot the joint probabilities of blob radial velocity versus vertical size, separately for the four gases. We find similar average vertical blob scales $\langle 2 a\rangle \sim 5 \mathrm{~cm}$ in all four plasmas, consistent with blobs forming from the wave crest of an interchange wave with same vertical wavelength $\approx 13 \mathrm{~cm}$. The average of $\delta n / n$ [see Fig. 2(f) for definition] is also similar in the four plasmas, lying between $0.69(\mathrm{He})$ and $0.76\left(\mathrm{H}_{2}\right)$. Clear differences are found, however, for the typical blob velocities, ranging between $\left\langle v_{\text {blob }}\right\rangle \sim 500 \mathrm{~m} / \mathrm{s}$ (Ar) and $\left\langle v_{\text {blob }}\right\rangle \sim$ $1700 \mathrm{~m} / \mathrm{s}(\mathrm{He})$.

In the following, we motivate a blob velocity scaling for the quasistationary phase of blob propagation and put it in the context of earlier published scaling laws. We start from $\nabla \cdot \mathbf{J}=0$ (quasineutrality). Assuming a constant electron temperature over the blob and $T_{i} \ll T_{e}$ for the electron heated TORPEX plasma [22], and integrating $\nabla \cdot \mathbf{J}=0$ along the magnetic field, thus assuming a 2D structure of the blob, we find $[14,23]$

$$
\frac{2 c_{s}^{2} m_{i}}{R B} \frac{\partial n}{\partial y}=\frac{n m_{i}}{B^{2}} \frac{D}{D t} \nabla^{2} \phi-\frac{n e^{2} c_{s}}{T_{e} L} \tilde{\phi}+\frac{n m_{i}}{B^{2}} \nu_{i n} \nabla^{2} \phi .
$$

Here, $c_{s} \approx \sqrt{T_{e} / m_{i}}$ is the ion sound speed, $\phi$ the electrostatic plasma potential, and $\tilde{\phi}=\phi-\phi_{f}$ the deviation from its floating value $\phi_{f} \approx 3 T_{e} / e$ [24]. $\nu_{\text {in }}$ is the ionneutral collision frequency and $D / D t=\partial / \partial t+\mathbf{v}_{\mathbf{E} \times \mathbf{B}} \cdot \nabla$. The term on the left-hand side is the divergence of the electron diamagnetic current, the drive for the blob motion. The current loop is closed by the ion polarization current, sheath currents, and the ion current caused by a neutral friction force. These effects are taken into account by the three terms on the right-hand side of Eq. (1). We note here that for $\alpha \ll 1$, where $\alpha$ is the incidence angle between

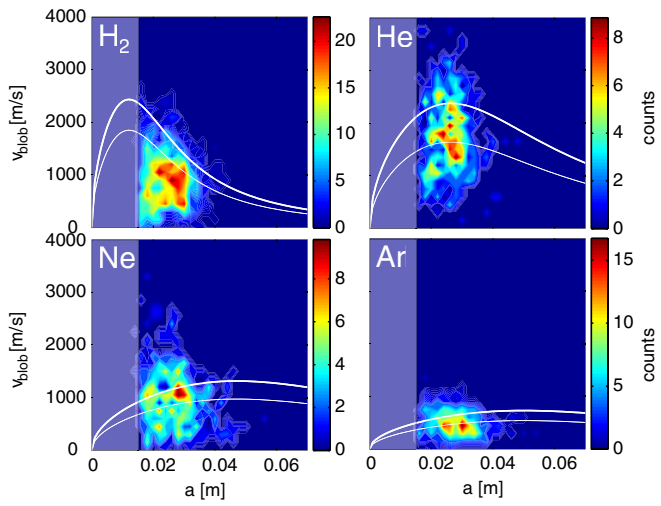

FIG. 3 (color online). Joint probabilities of measured radial blob velocity versus vertical blob size in the 4 plasmas. The scaling law Eq. (2) for $\nu_{\text {in }}=0$ is superimposed (thick solid) for $\delta n / n=1$ and (thin solid) for $\delta n / n=0.76,0.69,0.74,0.74\left(\mathrm{H}_{2}\right.$, $\mathrm{He}, \mathrm{Ne}, \mathrm{Ar})$. Shaded areas show the range of blob sizes that are not accessible experimentally. magnetic field lines and the limiter $(\alpha \approx \pi / 2$ in the present experiment), we expect a contribution of the electron diamagnetic current to blob parallel currents of the order of $T_{e} /(\alpha B)(\partial n / \partial y)$ [20]. For the hydrogen blobs, this becomes significant for $\alpha \lesssim 0.2$.

We now estimate the magnitude of the different terms in Eq. (1). We assume that the density blob is a monopole structure and the potential blob a dipole with a radially elongated positive and negative pole on the top and on the bottom side of the blob, respectively. This picture agrees with numerical simulations [11]. We estimate the terms of Eq. (1) at the position of the positive pole of the potential as follows: $\partial n / \partial y \sim-\delta n / a$, similarly to [7] $D / D t \sim$ $\sqrt{2} c_{s} / \sqrt{R a}$ (ideal interchange growth rate), $\nabla^{2} \phi \sim$ $-\tilde{\phi} / a^{2}$, and $\tilde{\phi} \sim B v_{\text {blob }} a$. We then obtain

$$
v_{\text {blob }}=\frac{\sqrt{\frac{2 a}{R}} c_{s}}{1+\frac{1}{\rho_{s}^{2} L} \sqrt{\frac{R}{2}} a^{5 / 2}+\frac{\nu_{\text {in }} \sqrt{R a}}{\sqrt{2} c_{s}}} \frac{\delta n}{n},
$$

where $\rho_{s} \approx \sqrt{T_{e} m_{i}} /(e B)$ is the ion sound Larmor radius. The three terms in the denominator represent the divergence of the ion polarization current, the sheath current, and the ion current due to neutral friction, respectively. The factor $\delta n / n$ describes the slowing down by a finite background density [13]. In the limit where sheath losses and ion-neutral collisions are negligible, i.e., if $\tilde{a}^{5 / 2}, \tilde{\eta} \sqrt{\tilde{a}} \ll 1$ (see below for definitions), we obtain $v_{\text {blob }}=\sqrt{2 a / R} c_{s}$ similar to [7,8]. If sheath losses become dominant $\left(\tilde{a}^{5 / 2} \gg\right.$ $1, \tilde{\eta} \sqrt{\tilde{a}})$, we find the scaling $v_{\text {blob }}=2 \frac{L}{R} \frac{\rho_{s}^{2}}{a^{2}} c_{s}$ of [6]; when ion-neutral friction dominates $\left(\tilde{\eta} \gg 1 / \sqrt{\tilde{a}}, \tilde{a}^{2}\right)$, we retrieve $v_{\text {blob }}=2 c_{s}^{2} /\left(R \nu_{\text {in }}\right)$, which is experimentally verified in [4].

In Fig. 3, the scaling provided by Eq. (2) is drawn together with the experimental data, showing good agreement for the four gases. We assume a typical blob temperature of half the peak value of the temperature profiles measured with LPs, i.e., $T_{e}=4,7,6$, and $3.5 \mathrm{eV}$ for $\mathrm{H}_{2}$, $\mathrm{He}, \mathrm{Ne}$, and $\mathrm{Ar}$, respectively, and we neglect ion-neutral collisions by setting $\nu_{\text {in }}=0$.

To investigate the parameter regime covered by the ion mass scan, we now turn to adimensional units. Similarly to [9], we normalize the blob radial velocity and its vertical size to

$$
v_{\text {blob }}^{*}=\left(\frac{2 L \rho_{s}^{2}}{R^{3}}\right)^{1 / 5} c_{s}, \quad a^{*}=\left(\frac{4 L^{2}}{\rho_{s} R}\right)^{1 / 5} \rho_{s} .
$$

For this case, the dimensionless radial blob velocity $\tilde{v}_{\text {blob }}$ and the dimensionless vertical blob size $\tilde{a}$ are linked by

$$
\tilde{v}_{\text {blob }}=\frac{\sqrt{2 \tilde{a}} \delta n / n}{1+\sqrt{2} \tilde{a}^{5 / 2}+\tilde{\eta} \sqrt{\tilde{a}}}, \quad \tilde{\eta}=\frac{\nu_{\text {in }} \rho_{s}}{c_{s}}\left(\frac{L R^{2}}{\sqrt{8} \rho_{s}^{3}}\right)^{1 / 5} .
$$

In Fig. 4, we plot the data using this normalization. We superimpose the curve described by Eq. (4) for $\tilde{\eta}=0$ and 


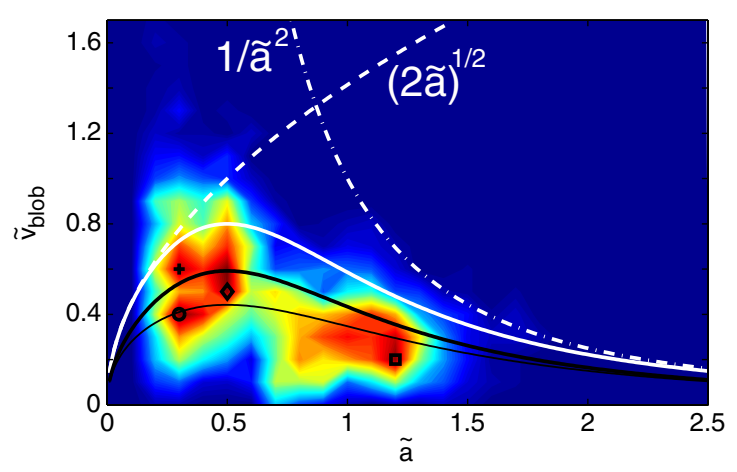

FIG. 4 (color online). Joint probability of $\tilde{v}_{\text {blob }}$ versus $\tilde{a}$. For better visibility, the distribution for $\mathrm{H}_{2}(\square)$ and $\mathrm{He}(\diamond)$ are normalized to 1 , the ones of $\mathrm{Ne}(+)$ and $\mathrm{Ar}(\bigcirc)$ to 0.5 . The symbols indicate the peak of each distribution. The scaling law Eq. (4) for $\tilde{\eta}=0, \delta n / n=0$ (white curve), $\tilde{\eta}=0, \delta n / n=0.73$ (thick black curve), and $\tilde{\eta}=0.6, \delta n / n=0.73$ (thin black curve), as well as the $1 / \tilde{a}^{2}$ and $\sqrt{2 \tilde{a}}$ limits, are indicated.

$\delta n / n=0$ (white curve) and $\delta n / n=0.73$ (thick black curve). The dashed line is the expected blob velocity in the absence of parallel currents $[7,8]$ and the dash-dotted line the scaling where sheath currents are dominant [6]. We find $\tilde{a}$ of the order of 1 . By varying the ion mass, both regimes $\tilde{a}<1$ and $\tilde{a}>1$ are obtained. For $\tilde{a}<1$, the parallel currents play a minor role and the dashed line approaches the experimental results. However, we observe strong deviations from this scaling for $\tilde{a}>1$, interpreted as due to the growing importance of parallel currents. Only in this range does the $1 / \tilde{a}^{2}$ scaling show quantitative agreement with experimental findings.

Thus far we have assumed $\nu_{\text {in }}=0$. We provide now an estimate of an upper bound of $\tilde{\eta}$ in Eq. (4). With the measured neutral background pressures $p_{n} \approx 0.017,0.02$, 0.014 , and $0.0085 \mathrm{~Pa}$ for $\mathrm{H}_{2}, \mathrm{He}, \mathrm{Ne}$, and $\mathrm{Ar}$, respectively, a momentum transfer cross section of $\sigma^{\mathrm{mt}} \leqslant 2 \times 10^{-18} \mathrm{~m}^{2}$ [25], an ambient temperature $T_{\mathrm{amb}}$ of $0.025 \mathrm{eV}$, and an upper bound for the ion temperature $T_{i} \lesssim 1 \mathrm{eV}$, we obtain from $\nu_{\text {in }}=\frac{p_{n}}{T_{\mathrm{amb}}} \sigma^{\mathrm{mt}} \boldsymbol{v}_{t h, i}$ values of $\tilde{\eta} \approx 0.5-0.6$ for all four gases. By setting $\tilde{\eta}=0.6$ in Eq. (4), a relatively small difference $(\leq 25 \%)$ is obtained compared to the case $\tilde{\eta}=$ 0, as shown in Fig. 4. This indicates that blob velocity is only weakly reduced by ion-neutral collisions.

In conclusion, cross-field blob velocity and size have been investigated experimentally in TORPEX in an open magnetic field line configuration. This features constant curvature along the field lines, nearly constant connection length, and almost perpendicular incidence of the magnetic field on the wall. Large blob statistics over a wide parameter range allowed a quantitative comparison with a blob velocity scaling, also derived here, which agrees well with the whole experimental data set. In the absence of ionneutral collisions, it recovers the commonly used SOL scalings $\tilde{v}_{\text {blob }} \propto 1 / \tilde{a}^{2}[6]$ and $\tilde{v}_{\text {blob }} \propto \sqrt{\tilde{a}}[7,8]$ for $\tilde{a} \gg 1$ and $\tilde{a} \ll 1$, respectively, where $\tilde{a}$ is the normalized vertical blob scale. Ion-neutral collisions become the dominant damping term for blob velocity when the adimensional parameter $\tilde{\eta}$ satisfies $\tilde{\eta} \gg 1 / \sqrt{\tilde{a}}$, $\tilde{a}^{2}$. In this limit we retrieve the scaling presented in [4]. We have obtained two regimes of blob motion in the experiment. In the regime $\tilde{a}<1$, blob velocity is mainly damped by crossfield ion polarization currents. For $\tilde{a}>1$, it is limited by parallel currents to the sheath. The damping due to ionneutral collisions is found to be weak $(\leq 25 \%)$.

This work is partly supported by the Fonds National Suisse de la Recherche Scientifique. The authors thank B.P. Duval and F. M. Poli for the careful reading of the manuscript.

[1] S. J. Zweben, Phys. Fluids 28, 974 (1985); J. A. Boedo et al., Phys. Plasmas 8, 4826 (2001); J. L. Terry et al., Phys. Plasmas 10, 1739 (2003); R. Sánchez et al., Phys. Rev. Lett. 90, 185005 (2003); M. Spolaore et al., Phys. Rev. Lett. 93, 215003 (2004).

[2] S. H. Müller et al., Phys. Plasmas 14, 110704 (2007).

[3] I. Furno et al., Phys. Rev. Lett. 100, 055004 (2008).

[4] N. Katz et al., Phys. Rev. Lett. 101, 015003 (2008).

[5] G. Y. Antar et al., Phys. Rev. Lett. 87, 065001 (2001); T. A. Carter, Phys. Plasmas 13, 010701 (2006).

[6] S. I. Krasheninnikov, Phys. Lett. A 283, 368 (2001).

[7] O. E. Garcia et al., Phys. Plasmas 12, 090701 (2005).

[8] J. R. Myra and D. A. D'Ippolito, Phys. Plasmas 12, 092511 (2005); see TABLE III (Local 2D blob).

[9] G. Q. Yu and S. I. Krasheninnikov, Phys. Plasmas 10, 4413 (2003).

[10] A. Y. Aydemir, Phys. Plasmas 12, 062503 (2005).

[11] O. E. Garcia et al., Phys. Plasmas 13, 082309 (2006).

[12] W. Fundamenski, W. Sailer, and JET EFDA contributors, Plasma Phys. Controlled Fusion 46, 233 (2004); A. Schmid et al., Plasma Phys. Controlled Fusion 50, 045007 (2008).

[13] J. R. Myra et al., Phys. Plasmas 13, 092509 (2006).

[14] S. I. Krasheninnikov et al., J. Plasma Phys. 74, 679 (2008).

[15] A. Fasoli et al., Phys. Plasmas 13, 055902 (2006).

[16] S. H. Müller et al., Phys. Plasmas 13, 100701 (2006).

[17] B. Labit et al., Phys. Rev. Lett. 98, 255002 (2007).

[18] M. Podestà et al., Phys. Rev. Lett. 101, 045001 (2008).

[19] C. Theiler et al., Phys. Plasmas 15, 042303 (2008).

[20] R.H. Cohen and D. D. Ryutov, Phys. Plasmas 2, 2011 (1995).

[21] S. H. Müller et al., Phys. Plasmas 12, 090906 (2005).

[22] M. Podestà et al., Plasma Phys. Controlled Fusion 47, 1989 (2005).

[23] D. A. D’Ippolito et al., Phys. Plasmas 9, 222 (2002).

[24] P. C. Stangeby, The Plasma Boundary of Magnetic Fusion Devices (Institute of Physics, Berkshire, 2000).

[25] A. E. Glassgold et al., Astrophys. J. 621, 808 (2005); A. V. Phelps, J. Appl. Phys. 76, 747 (1994). 